\title{
QUANTUM DOTS — THEORY FOR EXPERIMENTS
}

\author{
L. JACAK ${ }^{a}$, J. KRASNYJ ${ }^{b}$ AND A. Wójs ${ }^{a}$ \\ anstitute of Physics, Technical University of Wrocław \\ Wybrzeże Wyspiańskiego 27, 50-370 Wroclaw, Poland \\ ${ }^{b}$ Institute of Mathematics, University of Opole, Oleska 48, 45-951 Opole, Poland
}

A simple model based on the effective-mass method and treating a quantum dot as a small irregularity of the periodic crystal field is developed and used for the description of the radiative recombination of an exciton captured in that quasi-zero-dimensional structure. The additional peaks appearing in the photoluminescence spectra at the critical quantum dot size are predicted as a consequence of the metastable excited states occurring in the energy spectrum of a confined exciton. The obtained dependence of the photoluminescence spectrum on the dot size and magnetic field reproduces well the a vailable experimental results.

PACS numbers: 73.20.Dx, 71.35.Ji, 78.20.Ls

\section{Introduction}

Quantum dots are the quasi-zero-dimensional semiconductor structures which can bind a small and well controllable number of carriers (conduction electrons and/or valence holes), and thus often referred to as artificial atoms [1]. A number of techniques have been mastered to obtain the dots. The lithography combined with etching allows for the creation of tiny pillars etched out of a quantum well structure, where the confinement of carriers comes from the nonzero electron affinity of the well material. The etched dots, typically rectangular or circular, can reach the diameters of $10 \div 100 \mathrm{~nm}$ [2]. In another method the miniature electrodes can be lithographically created above the surface of a quantum well. These electrodes produce an electric lateral confinement free of edge defects $[3,4]$. The confining barriers localizing the quasi-two-dimensional electrons can also be created by the focused-laser-beam (FLB) induced local interdiffusion of atoms between a pair of coupled quantum wells [5]. More recently various self-organization processes have been successfully employed in the spontaneous growth of the so-called self-assembled quantum dots (SAD). Such dots are spontaneously formed in the strain-induced Stranski-Krastanow transition, in the growth of materials with a significant mismatch of lattice constants [6-8]. The SAD's grow in the form of lenses or pyramids embedded in the substrate material, and typically reach $10-30 \mathrm{~nm}$ in diameter. 
What allows for a common treatment of different quantum dots, created by numerous growth techniques, is that they are often in form of small islets embedded in a surrounding crystal structure, typically in a quantum well structure. It seems that specially the optical experiments on quantum dots show their similar behaviour, which should be thus explained within a simple and general model. Namely, in a series of recent experiments carried out on various types of dots, the photoluminescence (PL) spectrum probing the recombination of excitons localized in the dots consisted of a couple of peaks, unlike it is in the case of the single-mode emission from quantum wells [5, 7-9]. As reported, the measured splitting is sensitive to the dot diameter. The application of a weak magnetic field leads to the emergence of the additional third PL peak [10], which however disappears in high fields, where only a single main PL peak is observed.

Due to the expected applications of quantum dots in the new-generation optical or opto-electronic devices, such as the quantum-dot-based lasers [11] or optical memories [12], the need arises to fully understand the optical properties of these systems. A number of attempts have already been made where the dot was modeled through its own band structure, different from that of the surrounding material [13]. In this paper we shall use a simple and general model derived from the effective-mass method, where the higher peaks in the PL spectrum appear and vanish together with the low-energy metastable states in the exciton energy spectrum. We must however limit ourselves here to the brief overview of the calculus and to the discussion of the most important results, while the complete solution of the presented model will be published elsewhere [14].

\section{Model}

Let us assume the model, where the dot is defined as a local perturbation to the crystal field of a surrounding semiconductor. Since the interaction between the quantum dot and the carriers (conduction electrons and valence holes) has the electric nature, the empty dot can either attract electrons and at the same time repel holes, or vice versa (obviously, also the charge-neutral electron-hole pairs can be localized in such a dot). In the following the dot-carrier interaction will be modeled by a pair of single-particle potentials different only in the sign: $\mp V_{0} \exp \left(-r^{2} / L^{2}\right)$, where $L$ measures the dot radius, and $V_{0}$ scales the interaction strength. In order to fix the signs we choose the dot binding electrons, i.e. the signs "-" and "+" in the above definition correspond to the electron and the hole, respectively. We shall also take into account the magnetic field $B$ acting across the quantum-dot plane, which rearranges the single-electron and single-hole energy spectra, and enhances the carrier confinement. The Hamiltonian $H$ of a confined electron-hole pair is a sum of the single-particle terms $H_{\mathrm{e}}$ and $H_{\mathrm{h}}$, and the Coulomb interaction potential $V_{\mathrm{C}}\left(r_{\mathrm{e}}, r_{\mathrm{h}}\right)=-e^{2} / \epsilon\left|r_{\mathrm{e}}-r_{\mathrm{h}}\right|$, scaled by the dielectric constant $\epsilon$. The single-electron Hamiltonian is

$$
H_{\mathrm{e}}=\frac{p_{\mathrm{e}}^{2}}{2 \mu_{\mathrm{e}}}+V_{\mathrm{e}}\left(r_{\mathrm{e}}\right)+\frac{1}{2} \hbar \omega_{\mathrm{ce}} l_{z \mathrm{e}},
$$

where $r_{\mathrm{e}}, p_{\mathrm{e}}$, and $l_{z \mathrm{e}}$ are electron position, momentum, and $z$-th component of angular momentum, $\mu_{\mathrm{e}}$ is the effective mass and $\omega_{c e}=e B / c \mu_{\mathrm{e}}$ is the cyclotron 
frequency. The single-hole Hamiltonian $H_{\mathrm{h}}$ is obtained from $H_{\mathrm{e}}$ by replacing the subscripts: $e \rightarrow h$, and reversing the sign of the angular-momentum term (due to the opposite charge). The bare single-particle potential of the electron is

$$
V_{\mathrm{e}}\left(r_{\mathrm{e}}\right)=-V_{0} \exp \left(-\frac{r_{\mathrm{e}}{ }^{2}}{L^{2}}\right)+\frac{1}{8} \mu_{\mathrm{e}} \omega_{\mathrm{ce}}^{2} r_{\mathrm{e}}^{2},
$$

and that of the hole $V_{\mathrm{h}}$ is again obtained from $V_{\mathrm{e}}$ by replacing the subscripts: $\mathrm{e} \rightarrow \mathrm{h}$, and reversing the sign of the first (bare confinement) term.

In the following we shall fix the curvature of the bare electron confinement, i.e. fix the following units of energy and length: $\hbar \omega_{0 \mathrm{e}}=$ const and $\lambda_{0 \mathrm{e}}=$ const, defined as: $\omega_{0 \mathrm{e}}^{2}=2 V_{0} / \mu_{\mathrm{e}} L^{2}$ and $\lambda_{0 \mathrm{e}}^{2}=\hbar / \mu_{\mathrm{e}} \omega_{0 \mathrm{e}}$, respectively. Varied will be the parameter $\alpha=\hbar \omega_{0 \mathrm{e}} / V_{0}=2 \lambda_{0 \mathrm{e}}^{2} / L^{2}$ (the inverse, $\alpha^{-1}$; counts the number of bound electron shells in the absence of a magnetic field).

\section{Electron-hole pair in the quantum dot}

Within the Hartree approach we shall look for the exciton wave-function in the form of a product: $\Phi\left(r_{\mathrm{e}}, r_{\mathrm{h}}\right)=\phi_{\mathrm{e}}\left(r_{\mathrm{e}}\right) \phi_{\mathrm{h}}\left(r_{\mathrm{h}}\right)$, where the electron and hole wave-functions $\phi_{\mathrm{e}}$ and $\phi_{\mathrm{h}}$ satisfy the usual self-consistent equations. The electron and hole Hartree Hamiltonians $\mathcal{H}_{\mathrm{e}}$ and $\mathcal{H}_{\mathrm{h}}$ are obtained by adding the self-consistent interaction terms to the single-particle IIamiltonians $H_{\mathrm{e}}$ and $H_{\mathrm{h}}$ defined by (1), i.e. by replacing the single-particle potentials $V_{e}$ and $V_{h}$ by the effective Hartree potentials $U_{\mathrm{e}}$ and $U_{\mathrm{h}}$ :

$$
U_{\mathrm{e}}\left(r_{\mathrm{e}}\right)=V_{\mathrm{e}}\left(r_{\mathrm{e}}\right)-\frac{e^{2}}{\epsilon} \int \mathrm{d} r_{\mathrm{h}} \frac{\left|\phi_{\mathrm{h}}\left(r_{\mathrm{h}}\right)\right|^{2}}{\left|r_{\mathrm{e}}-r_{\mathrm{h}}\right|}
$$

and $U_{\mathrm{h}}$ is obtained from $U_{\mathrm{e}}$ by the interchange of subscripts: $\mathrm{e} \leftrightarrow \mathrm{h}$. The electron and hole Hartree energies $E_{\mathrm{e}}$ and $E_{\mathrm{h}}$ are counted here from the bottom/top of the conduction/valence band, respectively, and the total exciton energy

$$
E=E_{\mathrm{e}}+E_{\mathrm{h}}-\frac{e^{2}}{\epsilon} \int \mathrm{d} r_{\mathrm{e}} \int \mathrm{d} r_{\mathrm{h}} \frac{\left|\phi_{\mathrm{e}}^{*}\left(r_{\mathrm{e}}\right)\right|^{2}\left|\phi_{\mathrm{h}}^{*}\left(r_{\mathrm{h}}\right)\right|^{2}}{\left|r_{\mathrm{e}}-r_{\mathrm{h}}\right|}
$$

is counted from the band-gap $E_{\mathrm{g}}$.

Provided that the Coulomb interaction is weaker than the electron confinement we solve the Hartree equations defined by $\mathcal{H}_{\mathrm{e}}$ and $\mathcal{H}_{\mathrm{h}}$ perturbatively. In the zeroth-order approximation we neglect the electron-hole interaction in Eq. (3) and approximate $V_{\mathrm{e}}$ by a harmonic well of frequency $\tilde{\omega}_{\mathrm{ce}}^{2}=\omega_{0 \mathrm{e}}^{2}+\omega_{\mathrm{ce}}^{2} / 4 \equiv \omega_{0 \mathrm{e}}^{2} \beta^{2}$. In this approximation, the electron ground-state energy is $E_{\mathrm{e}}=-V_{0}+\hbar \tilde{\omega}_{\mathrm{ce}}=$ $\hbar \omega_{0 \mathrm{e}}(-1 / \alpha+\beta)$, and the corresponding wave-function is a usual Gaussian.

In the next step of perturbation we put the expression for the zeroth-order electron wave-function into $U_{\mathrm{h}}$ (cf. Eq. (3)), and arrive at the eigenequation for $\mathcal{H}_{\mathrm{h}}$, with the potential $U_{\mathrm{h}}$ reading

$U_{\mathrm{h}}(r)=V_{0} \exp \left(-\frac{r^{2}}{L^{2}}\right)+\frac{1}{8} \frac{\mu_{\mathrm{e}}^{2}}{\mu_{\mathrm{h}}} \omega_{\mathrm{ce}}^{2} r^{2}-\frac{e^{2}}{2 \lambda_{0 \mathrm{e}}^{2}} \sqrt{\pi \beta} \exp \left(-\frac{\beta r^{2}}{2 \lambda_{0 \mathrm{e}}^{2}}\right) I_{0}\left(-\frac{\beta r^{2}}{2 \lambda_{0 \mathrm{e}}^{2}}\right)$,

where $I_{0}$ is the Bessel function. The typical shape of $U_{\mathrm{h}}$ is shown in Fig. 1. It has two minima, one at $r_{\mathrm{Lh}} \equiv 0$ and the other at $r_{\mathrm{Rh}}>0$. With the increase in $\alpha$, the energies of the pair of minima, $U_{\mathrm{Lh}}=U_{\mathrm{h}}(0)$ and $U_{\mathrm{Rh}}=U_{\mathrm{h}}\left(r_{\mathrm{Rh}}\right)$, decrease. 


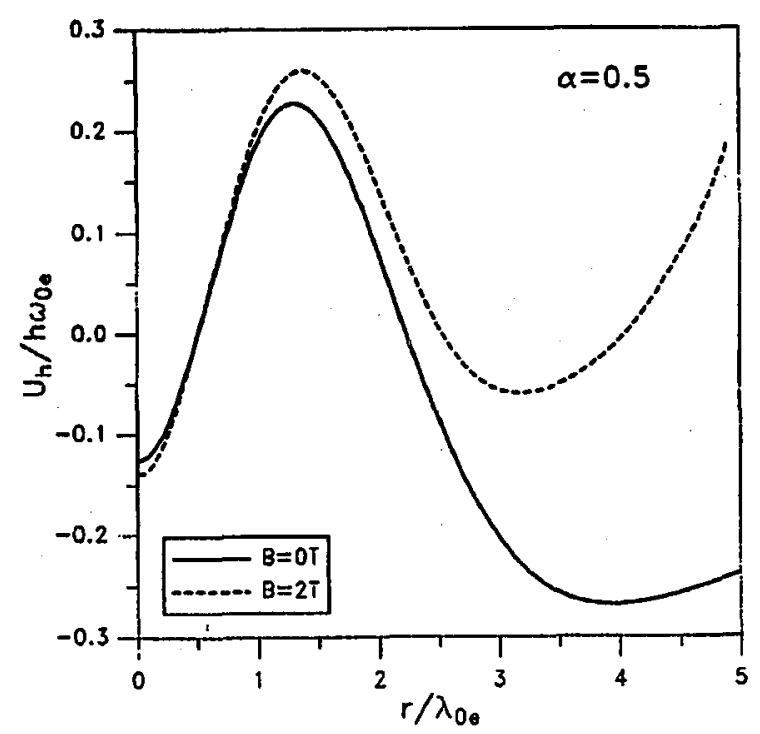

Fig. 1. Hole self-consistent potential $U_{\mathrm{h}}$ at $B=0$ (solid line) and at $B=2 \mathrm{~T}$ (dashed line), for $\hbar \omega_{0 \mathrm{e}}=7.5 \mathrm{meV}$ and $\alpha=0.5$.

Simultaneously, the second minimum approaches the first one and eventually disappears.

The motion of a hole in a double-well potential $U_{\mathrm{h}}$ can be solved approximately by independently studying the motions in the two wells, and later including (perturbatively) the coupling between the wells via the off-diagonal tunneling matrix element. Thus the pair of lowest-energy hole states can be obtained by diagonalizing $\mathcal{H}_{\mathrm{h}}$ in the two-dimensional subspace spanned by the pair of ground states in the two wells treated separately: $\psi_{\mathrm{Lh}}$ and $\psi_{\mathrm{Rh}}$. The ground state $(i=1)$ and the first excited state $(i=2)$ of the hole are hence assumed in the form

$$
\phi_{\mathrm{h}}{ }^{(i)}(r)=c_{\mathrm{L}}{ }^{(i)} \psi_{\mathrm{Lh}}(r)+c_{\mathrm{R}}{ }^{(i)} \psi_{\mathrm{Rh}}(r),
$$

with the coefficients $c_{\mathrm{L}}$ and $c_{\mathrm{R}}$ calculated from the minimum energy condition. Due to the non-vanishing coupling between the wells, the corresponding pair of energies: $E_{\mathrm{h}}^{(1)}$ and $E_{\mathrm{h}}^{(2)}$, are always separated by a gap, even in the case of degeneracy of the single-well levels. Due to the same parity (zero angular momentum) of both single-well ground states $\psi_{\mathrm{Lh}}$ and $\psi_{\mathrm{Rh}}$, also the two coupled states $\phi_{\mathrm{h}}^{(1)}$ and $\phi_{\mathrm{h}}^{(2)}$ are of the same parity. First $(i=1)$ corresponds to the hole residing in the center of the dot (bound state only in a magnetic field) and the latter $(i=2)$ to the hole forming a ring surrounding the dot. Summarizing, in result of a double-well structure of the Hartree potential, the low-energy excited state appears in the hole energy spectrum, with the same parity as the ground state.

In the next step of perturbation we shall now calculate for the pair of hole states the corresponding effective electron IIartree potentials: $U_{\mathrm{e}}^{(1)}$ and $U_{\mathrm{e}}^{(2)}$ (cf. Eq. (3)). As shown in Fig. 2, the potential $U_{\mathrm{e}}^{(2)}$ corresponding to the hole in the ring-like state usually also has two minima, at $r=r_{\mathrm{Le}}^{(2)} \equiv 0$ and at $r=r_{\mathrm{Re}}^{(2)}>0$, 


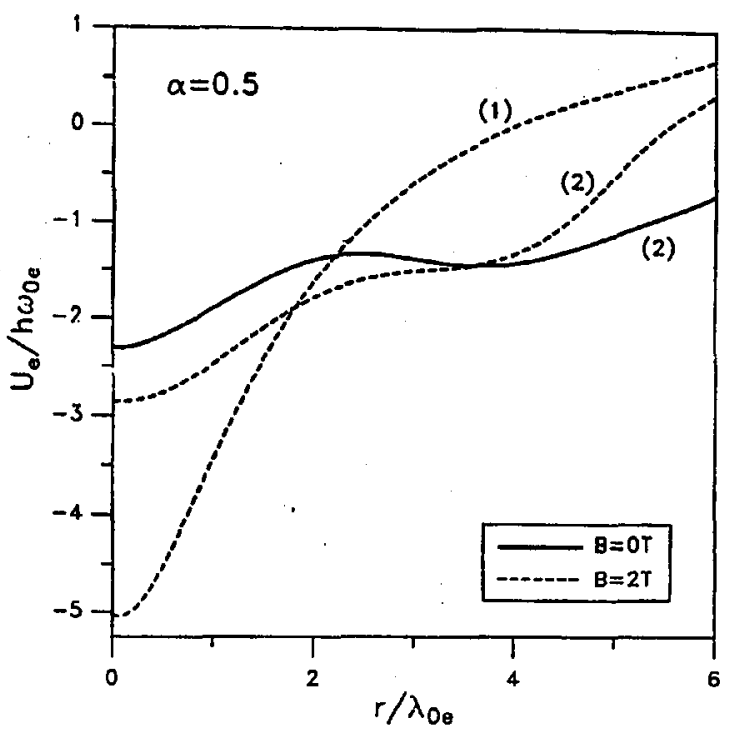

Fig. 2. Electron self-consistent potentials: $U_{\mathrm{e}}^{(2)}$ for $B=0$ (solid line), and $U_{\mathrm{e}}^{(1)}$ and $U_{\mathrm{e}}^{(2)}$ for $B=2 \mathrm{~T}$ (dashed lines), for $\hbar \omega_{0 \mathrm{e}}=7.5 \mathrm{meV}$ and $\alpha=0.5$.

while $U_{\mathrm{e}}^{(1)}$, corresponding to the hole in the center, always only one, at $r=r_{\mathrm{Le}}^{(1)} \equiv 0$.

Putting the potentials $U_{\mathrm{e}}^{(\mathrm{i})}$ into the IIartree Hamiltonian $\mathcal{H}_{\mathrm{e}}$ we arrive at the electron equation of motion, solved analogously as in case of the hole. Whenever $U^{(i)}$ has two minima, there will be two low-energy states with the same parity (zero angular momentum) in the electron energy spectrum, denoted as $\phi_{\mathrm{e}}{ }^{(i j)}(j=1$ and 2). These states are approximated in the form of linear combinations of the ground states of the two uncoupled wells

$$
\phi_{\mathrm{e}}^{(i j)}(r)=d_{\mathrm{L}}^{(i j)} \psi_{\mathrm{Le}}^{(i)}(r)+d_{\mathrm{R}}^{(i j)} \psi_{\mathrm{Re}}^{(i)}(r)
$$

In the state with $j=1$ the electron resides in the center of the dot, and in the state with $j=2$ occupies the ring around the second minimum of $U_{\mathrm{e}}$. Hence, depending on whether $U_{e}^{(i)}$ has one or two minima, there will be or not a low-energy excited electron state with the same parity as the ground state.

Tracing the binding and delocalization of electron and hole Hartree states in a varying dot size and magnetic field, and combining these states into possible pairs $(i, j=1,2)$, we can classify the possible states of an exciton

$$
\Phi^{(i j)}\left(r_{\mathrm{h}}, r_{\mathrm{e}}\right)=\phi_{\mathrm{h}}{ }^{(i)}\left(r_{\mathrm{h}}\right) \phi_{\mathrm{e}}^{(i j)}\left(r_{\mathrm{e}}\right)
$$

with energies

$$
E^{(i j)}=E_{\mathrm{h}}^{(i)}+E_{\mathrm{e}}^{(i j)}+V_{\mathrm{C}}^{(i)} .
$$

The Coulomb energy $V_{\mathrm{C}}{ }^{(i)}$ is here approximated by the expectation value of the Coulomb potential in the exciton state with the hole in its state $i$, and the electron in its zeroth-order state (in this approximation the interaction energy can be evaluated analytically). 
The crucial property of the states (8) is that all appearing electron and hole Hartree states have the same (zero) angular momentum, and therefore the dipole-type radiative transitions between each pair of levels are forbidden. Hence, when the relaxation through the emission of phonons is suppressed due to the small dot dimensions [9], and the only remaining mechanism of relaxation involves the emission of a far infrared photon, these excited states (8) are metastable, and give rise to the appearance of additional peaks in the PL spectra of quantum dots, as explicitly listed below.

In zero or very weak magnetic fields, there are two exciton states, with $(i j)=(21)$ and $(22)$, and thus there are two PL peaks separated by $\Delta E^{(21,22)}=$ $\left|E_{\mathrm{e}}^{(22)}-E_{\mathrm{e}}^{(21)}\right|$. The dependence of the spectrum on the dot size, consistent with a number of experiments $[5,7,8]$, is shown in Fig. 3.

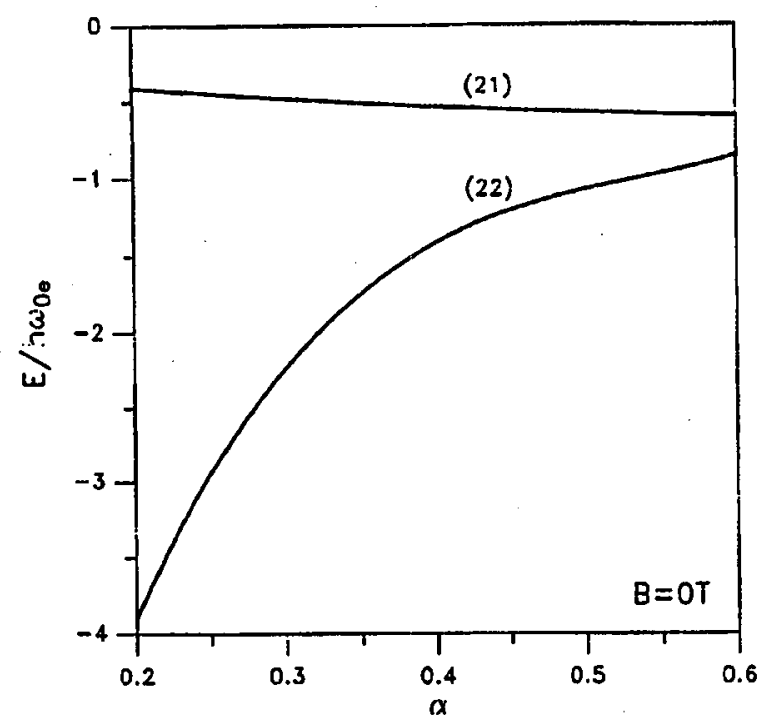

Fig. 3. Exciton recombination energies in the absence of a magnetic field: $E^{(22)}$ and $E_{(21)}$, for $\hbar \omega_{0 e}=7.5 \mathrm{meV}$, as a function of the parameter $\alpha$ connected with the dot radius (higher $\alpha$ corresponds to a smaller dot).

In relatively weak magnetic fields $(B \approx 2 \div 3 \mathrm{~T})$, there are two electron-hole pair states with $(i j)=(11)$ and $(21)$, and thus two PL peaks separated by a gap of $\Delta E^{(11,21)}=\left|E_{\mathrm{h}}^{(11)}-E_{\mathrm{e}}^{(21)}+V_{\mathrm{C}}^{(1)}-V_{\mathrm{C}}^{(2)}\right|$.

In moderate magnetic fields $(B \approx 4 \div 6 \mathrm{~T})$ and for certain range of the parameter $\alpha$, in addition to the doublet of states with $(i j)=(11)$ and $(21)$, the third electron-hole pair state appears with $(i j)=(22)$. Thus the third PL peak emerges, separated from the second by $\Delta E^{(21,22)}=\left|E_{\mathrm{e}}^{(22)}-E_{\mathrm{e}}^{(21)}\right|$. When $\alpha$ is either too small or too large, there triplet of peaks is replaced by a doublet, and further by a single peak for very small dots. Note that three is the maximum number of metastable states of a single exciton (PL peaks) predicted within our 
model at any magnetic field and dot size (at low excitation powers), which agrees with the experiment of Bayer et al. [10].

In strong fields $(B \geq 8 \mathrm{~T})$ higher peaks subsequently disappear, and the PL spectrum consists of a single main peak.

Summarizing, the magnetic-field evolution of the PL spectrum of a quantum dot qualitatively depends on the size of the quantum dot. Namely, the two scenarios are possible: (i) medium dot: there are two peaks at $B_{0}=0$, the third one emerges at $B_{1}>0$ and disappears at $B_{2}>B_{1}$, and finally the second peak disappears at $B_{3}>B_{2}$; (ii) small or large dot: there are two peaks for all fields below the critical value, at which the higher peak disappears.

\section{Conclusion}

In conclusion, the recombination of an exciton confined in a quantum dot has been studied theoretically with and without the inclusion of a magnetic field. The electron and hole self-consistent potentials, determined in the effective-mass approach, exhibit a double-well structure. The positions and depths of the pair of minima of these potentials depend strongly on the dot size. In consequence, the blueshift of the overall PL spectrum is observed with diminishing the dot. Moreover, the doublet of peaks corresponding to the occurrence of an exciton metastable state behaves exactly as observed in the experiment, when the dot radius is varied: (i) when the dot is of a medium size, the intensity of the additional peak is comparable to that of the main peak, and the energy gap between the two peaks is small, (ii) when the dot is smaller or larger than the intermediate, critical size, the additional peak is much weaker than the main peak, and the energy spacing between the two is large.

The magnetic field has a significant influence on the shape of the effective potentials, as it modifies the confinement of both carriers. In weak fields the hole potential has a shape of the double well, but both corresponding electron potentials have only a single minimum, and therefore only two PL peaks are observed. For intermediate magnetic fields and for certain sizes of the dot an additional minimum appears in the electron potential, which leads to the appearance of an additional, third PL peak. In strong magnetic fields both potentials have the single-well structure, which reduces the PL spectrum to a single peak.

\section{Acknowledgment}

The authors thank S. Bujkiewicz and M. Korkusiński (TU Wrocław) for the help with numerical calculations. A. Wójs acknowledges the financial support from the scholarship of the Foundation for Polish Science.

\section{References}

[1] For recent reviews and references see: T. Chakraborty, Comments Condens. Matter Phys. 16, 35 (1992); M. Kastner, Physics Today 46, 24 (1993); R.C. Ashoori, Nature 379, 413 (1996).

[2] T. Demel, D. Heitmann, P. Grambow, K. Ploog, Phys. Rev. Lett. 64, 788 (1990).

[3] R.C. Ashoori, H.L. Störmer, J.S. Weiner, L.N. Pfeifer, K.W. Baldwin, K. West, Phys. Rev. Lett. 71, 613 (1993). 
[4] B. Meurer, D. Heitmann, K. Ploog, Phys. Rev. Lett. 68, 1371 (1992).

[5] K. Brunner, U. Bockelmann, G. Abstreiter, W. Walter, G. Böhm, G. Tränkle, G. Weiman, Phys. Rev. Lett. 69, 3216 (1992).

[6] P.M. Petroff, S.P. DenBaars, Superlallices Microstruct. 15, 15 (1994).

[7] S. Fafard, D. Leonard, J.L. Merz, P.M. Petroff, Appl. Phys. Lett. 65, 1388 (1994).

[8] S. Fafard, R. Leon, D. Leonard, J.L. Merz, P.M. Petroff, Phys. Rev. B 52, 5752 (1995).

[9] U. Bockelmann, K. Brunner, G. Abstreiter, Solid State Electron. 37, 1109 (1994).

[10] M. Bayer, A. Schmidt, A. Forchel, F. Faller, T.L. Reinecke, P.A. Knipp, A.A. Dremin, V.D. Kulakovskii, Phys. Rev. Lett. 74, 3439 (1995).

[11] N. Kirstaedter, N.N. Ledentsow, M. Grudmann, D. Bimberg, V.M. Ustinov, S.S. Ruvimov, N.V. Maximov, P.S. Kop'ev, Z.J. Alferov, V. Richter, P. Werner, V. Gösele, J.R. Jenreich, Electron. Lett. 30, 1416 (1994).

[12] K. Imamura, Y. Sugiyama, Y. Nakata, S. Muto, N. Yokoyama, Jpn. J. Appl. Phys. 34, L1445 (1995).

[13] A. Wójs, P. Hawrylak, S. Fafard, L. Jacak, Phys. Rev. B 54, 5604 (1996); A. Wójs,

P. Hawrylak, Solid State Commun. 100, 487 (1996).

[14] L. Jacak, J. Krasnyj, M. Korkusiński, A. Wójs, to be published. 